\title{
Renal Function of Living Kidney Donors at Kenyatta National Hospital Nairobi-Kenya
}

\author{
A.B. Ochwila, J.K. Kayima, S.O. Mcligeyo, A.J.O. Were
}

\begin{abstract}
:
Background: The outcome of live kidney donation has been of concern since the recognition of hyperfiltration injury post live kidney donation.

Studies on the topic have arrived at different conclusions regarding kidney function following live kidney donation; some suggest the possibility of renal progression while others report renal function similar to that of the general population. However, there are no studies done in a homogeneously African population to compare with.

Beginning the year 2010 at Kenyatta National Hospital, Kenya, live kidney donation happened regularly with an average of two live related kidney donation and transplantation per month.

The purpose of this study was to assess the impact of renal donation on renal function of live kidney donors at Kenyatta national hospital.

Objective: To determine serum creatinine levels, eGFR, prevalence of proteinuria, and hypertension among living kidney donors at Kenyatta National Hospital.

Study design: Cross-sectional descriptive study

Methods: Using a questionnaire, a targeted history was obtained from kidney donors. A venous blood sample was drawn for serum creatinine measurement from which an eGFR was calculated using Cockroft-Gault equation. A sample of urine was collected from which proteinuria was determined using a standard urinary dip stick. Furthermore, blood pressure, height and weight were measured followed by the determination of the body mass index of the study subjects. Patient's pre-nephrectomy records were reviewed and blood pressure, weight, height, serum creatinine levels were recorded. Their body mass index and estimated glomerular filtration rate pre-nephrectomy was then calculated. The prevalence of hypertension and proteinuria was expressed as proportions. Using a paired student's t-test, mean changes for serum creatinine, estimated glomerular filtration rate, diastolic blood pressure and systolic blood pressure were determined. Statistical significance was pegged at $P$-value of $<0.05$.

Results: A total of 52 subjects were enrolled in the study. The mean ( \pm standard deviation) estimated glomerular filtration rate post-nephrectomy was $79.96 \mathrm{mls} / \mathrm{min} / \mathrm{M}^{2}\left( \pm 31.95 \mathrm{mls} / \mathrm{min} / \mathrm{M}^{2}\right)$ which transformed to $85.62 \%$ of the pre-nephrectomy estimated glomerular filtration rate. The prevalence of proteinuria, and hypertension was $21.2 \%$ and $9.6 \%$ respectively. New onset hypertension was $6 \% .40 \%$ of the subjects were either overweight or obese. There was a significant mean change for serum creatinine, estimated glomerular filtration rate and diastolic blood pressure when pre-neprectomy and post-nephrectomy values were compared with $p$ values of $<0.0001,0.002$ and 0.008 respectively.

Conclusion: At the mean duration of follow up of 15.9 months, the kidney donors studied regained their renal function with a tendency towards hyperfiltration. The prevalence of proteinuria and hypertension was low. The results of the study compared well with those obtained from other studies.
\end{abstract}

\section{Introduction}

Chronic kidney disease (CKD) is a public health problem of increasing significance, consuming a growing proportion of healthcare resources (1).

The prevalence of end stage renal disease (ESRD) in developing countries is on the increase. This could be attributed to the growing incidence of risk factors for CKD namely diabetes mellitus, hypertension, HIV and chronic glomerulonephritis among others $(2 ; 3)$

Following ESRD, renal replacement therapy (RRT) becomes necessary for survival of patients. The available forms of RRT are hemodialysis, peritoneal dialysis and renal transplantation. Transplantation is the best mode of RRT as it is cost effective overall and is associated with less morbidity and mortality compared to dialysis; as a result it accords ESRD patients the best quality of life (4).

Sources of renal allograft include; cadaveric, living non-related and living related donors (5). The later is the most preferred since it is not associated with delayed graft function and minimal immunosuppression is required to stem off rejection and maintain graft function. 
In Kenya with a population of approximately 40 million, according to Kenya Renal Association (KRA), an estimated 6000 patients suffer kidney failure annually. Renal transplantation services are offered at Kenyatta National hospital (KNH); a public referral hospital and a few private hospitals. The first renal transplant was performed in 1978 following erroneous nephrectomy of a pelvic horse shoe kidney in a young man (6). Since then kidney transplants were sporadic and expensive with modest results. More recently, from the year 2010 an average of 2 live kidney donations occurs per month reflecting the life of the Interlife project; a public private partnership between KNH and Norvatis whose main goal is the development of a centre of excellence in kidney transplantation through training of health care professionals by recognized kidney transplant specialists from Spain with a focus on enhancing surgical techniques as well as increasing efficiency and reducing cost of transplants and access to drugs.

At $\mathrm{KNH}$, potential kidney donors are screened for compatibility with the recipient, occult kidney disease, co-morbidities commonly associated with kidney disease such as hypertension and diabetes and for suitability to undergo anesthesia and surgery. Thus, live kidney donors are highly selected and healthy individuals.

With the increasing burden of ESRD, many patients will require renal replacement therapy in the form of renal transplantation. There is therefore need for a corresponding increase in the number of living kidney donation to meet shortage of organs.

Data regarding kidney function in living donors is limited. This is because following renal donation, there are no standard guidelines for follow up of living related donors in most kidney transplantation centres. As a result, there is no universally accepted duration for follow up of kidney donors. In spite of this, most studies done so far suggest need for regular follow up.

In Africa, data regarding renal function in living renal donors is scanty. Two studies in S. Africa $(7,8)$ with predominantly white subjects have suggested stability of kidney function following live kidney donation. There are no studies in Kenya and the rest Africa regarding living kidney donors.

Recently, a prospective study done in United Kingdom (9), demonstrated a decline in renal function of donors and an increase in the incidence of new onset hypertension hence rekindling the debate on the safety of kidney donors.

\section{Materials And Methods}

Design: This was a Cross sectional descriptive study.

Study site: Kenyatta National Hospital $(\mathrm{KNH})$ renal unit. $\mathrm{KNH}$ is the principal public treatment centre for ESRD and the main national teaching and referral hospital.

Study population: All individuals who had had live donor nephrectomy at Kenyatta National Hospital in the period starting one week and beyond post nephrectomy.

The University of Nairobi Department of Medicine and Therapeutics and Kenyatta National Hospital/University of Nairobi Research and Ethics committee approved the study. Persons who met the inclusion criteria were contacted through their mobile Phone numbers available in their files or where this was not available, through their respective renal allograft recipients who are followed up regularly at the renal clinic. The reason for their being contacted was explained and those who agreed were given an appointment to come to $\mathrm{KNH}$ renal unit for review and possible recruitment. From the records available, 63 persons were eligible for recruitment. We traced 57 persons and 52 of those traced were included in the study. A total of 11 eligible persons who did not participate in the study were 4 males and 7 females. This was a population study hence all persons who met the inclusion criteria were recruited and studied.

Data collection: All persons studied signed a written informed consent form upon agreeing to participate in the study. The principal investigator then administered the study questionnaire by way of direct questioning. Information obtained included biodata, and post nephrectomy morbidity. The BP, weight and height were measured in the standard way using standardized measuring instruments. The BMI was then calculated, using the formula: $\mathrm{BMI}=$ weight $(\mathrm{KGs}) /$ height $\left(\mathrm{M}^{2}\right)$. All data collected was entered in the questionnaire.

The clinical variables: Hypertension was defined as a systolic blood pressure $\geq 140 \mathrm{mmHg}$ and /or diastolic blood pressure $\geq 90 \mathrm{mmHg}(10)$. Body mass index (BMI) (11) was used as a measure of total obesity : $<18.5$, underweight; $18.5-25$ normal; $>25$ overweight/obesity. eGFR was classified according to the KDOQI guidelines for classification of CKD (12).

Sample collection and analysis: Using a tourniquet, a suitable vein in the antecubital fossa was located and $2 \mathrm{mls}$ of venous blood was drawn after observing the universal safety precautions. The blood was dispensed in a clean labeled cuvette. A clean labeled poly pot was given to the participants to collect $5 \mathrm{mls}$ of midstream spot urine specimen. The samples were submitted to KNH renal laboratory for analysis.

Proteinuria was measured using a standard urine dip stick and the result was noted. 
Analysis for serum creatinine was done by direct calorimetric method using Technicon RA-1000 analyzer. eGFR was calculated using Cockroft-Gault equation.

Pre-nephrectomy records were reviewed and documented prenephrectomy data was extracted.

Quality assurance: All measurements and specimens collected were undertaken using standard instruments and techniques. In addition, standard operating procedures were followed in carrying out of laboratory analysis.

Data storage: All the raw data in this study was filed and stored in a lockable drawer accessible only to the principal investigator. In addition, all the sheets were checked to confirm completeness before being filed. Furthermore, the data was entered by the principal investigator into a pass word protected ms-excel data base.

Data presentation and analysis: Analysis was undertaken using the SPSS version 17.0 statistical software. Descriptive statistical analyses such as means, medians and standard deviations were generated for continuous variables and frequency tables were produced for categorical variables. Prevalence conditions were calculated as percentages within $95 \%$ confidence intervals. Associations between pre and post live kidney donation BP, eGFR and serum creatinine was made using paired student $t$-test. Statistical significance was defined as $p$-value of $\leq$ 0.05 .

\section{Results}

Between October 2011 and April 2012, 52 out of the expected 63 living kidney donors were studied. The mean age of kidney donation was 32.7 years (range: $21-50$ years). $51.9 \%$ were male, $67.3 \%$ were married, all had some form of formal education and $63.5 \%$ were in gainful employment. 11 were not studied, 7 female and 4 male.

Generally all patients enjoyed good physical health. Only one 60 year old woman used NSAIDS routinely as analgesia for the pain from osteoarthritis that had been prescribed for her in the orthopaedic clinic

Table 1: Clinical variables

\begin{tabular}{|c|c|c|c|c|}
\hline Variable & $\mathbf{n}$ & Mean (Range) & Median & $\begin{array}{l}\text { Standard } \\
\text { Deviation }\end{array}$ \\
\hline Duration post-nephrectomy (months) & 52 & $15.90(0.4-157)$ & 12.50 & 23.90 \\
\hline post-nephrectomy BMI & 52 & $24.40(17.4-34.9)$ & 23.65 & 4.13 \\
\hline \multirow{2}{*}{$\begin{array}{l}\text { Serum creatinine }(\boldsymbol{\mu m o l s} / \mathbf{l}): \\
\text { pre-nephrectomy } \\
\text { post-nephrectomy }\end{array}$} & 50 & $86.50(51-123)$ & 86.00 & 13.73 \\
\hline & 52 & $106.15(51-229)$ & 106.00 & 29.58 \\
\hline \multirow{2}{*}{ 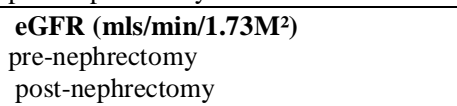 } & 50 & $93.39(53.2-150.4)$ & 90.09 & 21.34 \\
\hline & 52 & $80.25(37.1-186.5)$ & 70.55 & 31.91 \\
\hline
\end{tabular}

$\mathrm{M}^{2}$, square meters; $\mu$ mols, micromoles; 1 , liter; eGFR, estimated glomerular filtration rate; mls, milliliters; min, minute; BMI, body mass index

The duration post kidney donation ranged from a minimum of two weeks to a maximum of 15 years with a mean of 15.9 months. The mean BMI post kidney donation was 24.40 , standard deviation 4.13. There was a significant rise in serum creatinine level from a mean pre-nephrectomy level of $86.50 \mu \mathrm{mol} / 1$ to 106.15 $\mu$ mols/l post-nephrectomy. As a result a significant drop was observed in the eGFR from a mean prenephrectomy level of $93 \mu \mathrm{mols} / 1$ to $80.25 \mu \mathrm{mols} / 1$.

Table 2: eGFR pre- nephrectomy

\begin{tabular}{lcl}
\hline $\mathbf{e G F R}\left(\mathbf{m l s} / \mathbf{m i n} / \mathbf{m}^{2}\right)$ & $\mathbf{n}$ & $\mathbf{\%}$ \\
$<15$ & 0 & $0 \%$ \\
$15-29.999$ & 0 & $0 \%$ \\
$30-59.999$ & 3 & $6.0 \%$ \\
$60-89.999$ & 21 & $42.0 \%$ \\
$\geq 90$ & 26 & $52 \%$ \\
\hline
\end{tabular}

eGFR, estimated glomerular filtration rate; mls, milliliters; min, minute; $\mathrm{m}^{2}$, square metres

Table 3: eGFR post-nephrectomy

\begin{tabular}{llcl}
\hline Stage & eGFR(mls/Min/m $\left./ \mathbf{m}^{2}\right)$ & $\mathbf{n}$ & \% \\
$5($ ESRD) & $<15$ & 0 & $0 \%$ \\
4 & $15-29.999$ & 0 & $0 \%$ \\
3 & $30-59.999$ & 10 & $19.2 \%$ \\
2 & $60-89.999$ & 28 & $53.8 \%$ \\
1 & $\geq 90$ & 14 & $26.9 \%$ \\
\hline
\end{tabular}

eGFR, estimated glomerular filtration rate; mls, milliliters; min, minute; $\mathrm{m}^{2}$, square metres; ESRD, end-stage renal disease 
Majority of the kidney donors could be classified as CKD stage $2(54 \%)$ and stage 1 (27\%) post kidney donation.

Table 4: Prevalence of Proteinuria

\begin{tabular}{lll}
\hline Degree of proteinuria & $\mathbf{N}$ & \% \\
Nil & 41 & 78.8 \\
Trace $(15-30 \mathrm{mg} / \mathrm{dl})$ & 7 & 13.5 \\
$+(30-100 \mathrm{mg} / \mathrm{dl})$ & 3 & 5.8 \\
$++(100-300 \mathrm{mg} / \mathrm{dl})$ & 0 & 0 \\
$+++(300-1000 \mathrm{mg} / \mathrm{dl})$ & 1 & 1.9 \\
$++++(>1000 \mathrm{mg} / \mathrm{dl})$ & 0 & 0 \\
Total & $\mathbf{5 2}$ & $\mathbf{1 0 0}$ \\
\hline
\end{tabular}

Majority of patients with proteinuria had mild proteinuria with either trace or + . Only 1 had +++ , probably nephritic.

Table 5: Prevalence of hypertension

\begin{tabular}{llll}
\multicolumn{4}{c}{ Table 5: Prevalence of hypertension } \\
\cline { 2 - 4 } & & $\mathrm{N}$ & $\%$ \\
Prenephrectomy HTN & No & 48 & $96.0 \%$ \\
& Yes & 2 & $4.0 \%$ \\
Postnephrectomy HTN & No & 47 & $90.4 \%$ \\
& Yes & 5 & $9.6 \%$ \\
\hline
\end{tabular}

HTN, hypertension

Hypertension was defined as systolic blood pressure of $\geq 140 \mathrm{mmHg}$ and/or diastolic blood pressure of $\geq 90 \mathrm{mmHg}$. Hypertension was documented in $4 \%$ of the study subjects pre-nephrectomy. Post-nephrectomy, hypertension was observed in $9.6 \%$ of the population. There were three cases of new onset hypertension making $6 \%$ of the population. All cases were stage one hypertension.

Table 6: Mean change

\begin{tabular}{|l|l|l|l|l|}
\hline & & 95\% CI & \\
\cline { 3 - 4 } Variable & Mean & Lower & Upper & P Value \\
\hline Change in Serum creatinine $(\mu \mathrm{mol} / \mathrm{l})$ & 19.98 & 11.50 & 28.46 & $<0.0001$ \\
\hline Change in eGFR $(\mathrm{mls} / \mathrm{min})$ & -13.43 & -21.58 & -5.28 & 0.002 \\
\hline Change in BP Systolic $(\mathrm{mmHg})$ & 2.36 & -1.39 & 6.11 & 0.211 \\
\hline Change in BP Diastolic $(\mathrm{mmHg})$ & 4.10 & 1.12 & 7.08 & 0.008 \\
\hline
\end{tabular}

Significant mean change pre and post-nephrectomy was observed for serum creatinine, eGFR and diastolic blood pressure with $\mathrm{p}$ values of $<0.0001,0.002$, and 0.008 respectively.

Table 7: Post nephrectomy BMI

\begin{tabular}{llll}
\hline & $\mathbf{N}$ & $\mathbf{\%}$ \\
Underweight & $<18.5$ & 2 & 3.8 \\
Normal & $18.5-24.999$ & 29 & 55.8 \\
& & & \\
Overweight & $25-29.999$ & 14 & 26.9 \\
Obese & $\geq 30$ & 7 & 13.5 \\
Total & & $\mathbf{5 2}$ & $\mathbf{1 0 0}$ \\
\hline
\end{tabular}

$40.4 \%$ of the kidney donors were either overweight or obese post kidney donation

\section{Discussion}

In this cross-sectional descriptive study kidney donors generally did not report any ill health. None had been admitted in hospital or suffered any significant physical illness. Only one patient used non steroidal antiinflammatory drugs regularly for osteoarthritis.

The population was composed of mainly young kidney donors with a similar sex distribution. However, a total of 11 eligible persons did not participate in the study, 4 males and 7 females. Most donors who did not honor the appointment for participation cited feeling healthy and work commitments as the reason for their non participation. The mean duration post live kidney donation was 15.9 months reflecting the life of the Interlife project. 


\section{Serum creatinine and eGFR}

Studies have shown that following live kidney donation, renal adaptation is complete by one week ( 5 , $13,14)$ and GFR returns to $70-80 \%$ of the original.

A significant mean increase in serum creatinine with a corresponding decline in eGFR was observed. The study participants recovered $85.62 \%$ of the original 2 kidney function post kidney donation. $80.8 \%$ of the participants could be classified as CKD stage 1 and 2 with only $19.2 \%$ being classified as stage 3 . It is important to note that in the initial pre-nephrectomy work up of potential kidney donors isotopically determined GFR by DTPA method was used.

Wan et al (15) studied 72 patients and noted that at 1 year following kidney donation in his group of patients who had had isotopically determined GFR mean of $103.4 \mathrm{mls} / \mathrm{min}$ (SD $15.1 \mathrm{mls} / \mathrm{min}$ ) and serum creatinine $90.2 \mu \mathrm{mols} / 1$ ( SD $15.1 \mu \mathrm{mols} / \mathrm{l})$. One year later had mean serum creatinine levels of $118.6 \mu \mathrm{mols} / 1(\mathrm{SD}$ $19.9 \mu \mathrm{mols} / \mathrm{l})$ resulting in eGFR of $54.7 \mathrm{mls} / \mathrm{min} / 1.73 \mathrm{M}^{2}\left(\mathrm{SD} 9.26 \mu \mathrm{mols} / \mathrm{min} / 1.73 \mathrm{M}^{2}\right)$. Thus, $73 \%$ of the donors had eGFR $<60 \mathrm{mls} / \mathrm{min} / 1.73 \mathrm{M}^{2}$ equivalent to CKD stage 3.

Michael Siebels et al (13) observed that kidney function returned to73\% of the initial value of 2 kidneys after he followed up 166 patients for a mean of 38 months.

Nilay S. Patel et al (9) noted that at 1 year following live kidney donation, mean eGFR declined from $100 \mathrm{mls} / \mathrm{min} / 1.73 \mathrm{M}^{2}$ to $59 \mathrm{mls} / \mathrm{min} / 1.73 \mathrm{M}^{2}$. Thus, $53 \%$ of his patients were classified as CKD stage 3 or 4 . However he noted that kidney function remained stable thereafter.

Forcade et al (16) noted a lasting increase in GFR of the remaining kidney of $40 \%$ following donor nephrectomy.

The results of the study compares with those from the studies mentioned above such that an elevation in serum creatinine and consequently an overall decline in eGFR was demonstrated. An increase in one Kidney eGFR was also demonstrated. It is thought that the increase in GFR post nephrectomy is partly a consequence of hyperfiltration and intraglomerular hypertension emanating from tubule-glomerular and glomerulo-tubular balance adjustments which results in elaboration of the vasoconstrictors, angiotensin II and endothelin in the efferent glomerular artery $(5 ; 10)$. The study demonstrated a higher mean eGFR compared to other studies which may indicate a higher degree of hyperfiltration in the studied patients. This could be explained partly by the higher prevalence of overweight and obesity in our patients of $40.4 \%$. The Cockroft-Gault equation was used to estimate the GFR; the formula results are weight dependent hence the possibility of overestimating GFR in individuals with a big weight.

Of the participants with low eGFR post-nephrectomy one was a 60 year old who had donated a kidney 15 years prior hence the possibility of the natural loss of nephrons with advancing age could probably explain. Also, some of the participants had a low weight hence the risk of underestimating the GFR since the formula used is weight dependent. However, majority did not have anything significant observed that could explain the low eGFR.

\section{Proteinuria and microalbuminuria}

A prevalence of proteinuria of $21.2 \%$ that was mainly mild was observed.

Massimo Gai et al (17), in their review of studies that have assessed kidney function in live kidney donors, noted a proteinuria prevalence of $20 \%$ and albuminuria prevalence of $30-40 \%$.

Forcade et al (12) noted microalbuminuria in a few patients while Michael siebels et al (10) noted a low incidence of proteinuria mainly in elderly patients.

The prevalence of proteinuria in the study compares with that of Massimo Gai et al (17). It is possible that the hyperfiltration observed in the study subjects could have contributed to the pathophysiology of proteinuria. No evidence of urinary tract infection was noted on the urine dip stick examination of the participants who had proteinuria. It has been postulated that persistent intraglomerular hypertension results in subtle injuries to the glomeruli with consequent increase in protein leakage into the tubular fluid (5).

\section{Hypertension}

The prevalence of hypertension of $9.6 \%$ and new onset hypertension of $6 \%$ was observed.

Nilay S. Patel et al (9) diagnosed new onset hypertension in $10 \%$ of his study subjects at one year postnephrectomy.

Massimo Gai et al (14) noted no significant difference in hypertension between live kidney donors and the general population.

Abdu et al (8) in South Africa documented the prevalence of hypertension in live kidney donors of $24 \%$, range duration post-nephrectomy of 1-16 years.

In a similar setting as the study, unpublished studies by Hassan and Njau for their Masters of Medicine in Internal Medicine dissertation found the prevalence of hypertension in the general population at $12.6 \%$ and 
$13 \%$ respectively. The lower prevalence in the study compared to that of the general population could be due to the fact that the kidney donors were highly selected, young and healthy group before kidney donation.

\section{Strengths and limitations}

The strengths of the study are that to the best of our knowledge this is the first study to evaluate renal function among kidney donors in our setting. Thus, the results of this study may be used as a bench mark for future studies on the subject matter. Furthermore, to our knowledge, the study is the first to study a homogeneously African population by race hence it gives the first insight regarding renal function of the black African kidney donors.

The limitations of the study include the fact that GFR was not determined isotopically using the DTPA method hence the GFR is an estimate. In addition, it was not possible to trace and study all eligible kidney donors; as a result it is not possible to tell whether their inclusion would have altered the results. Also the population studied was homogeneously African hence extrapolation of the results obtained from this study to other races may not be possible.

In conclusion, post-nephrectomy the donors regained renal function with a tendency towards hyperfiltration. Serum creatinine increased significantly and there was a corresponding significant decline in eGFR. The prevalence of proteinuria was low but compared well with those obtained in other studies. The prevalence of hypertension was low. The results of the study compares with those obtained in other studies.

In view if the results obtained from the study, protocol follow up of live kidney donors, for example 6 monthly to help establish the general trend of kidney function following live kidney donation was recommended. Further, controlled studies on this group of subjects needed to be carried out in order to establish factors that may be associated with the outcome of kidney function following live kidney donation. In addition, subtle changes observed in the form of single kidney hyperfiltration, proteinuria and new onset hypertension need to be monitored. Also quantitative tests for urinary protein to be carried out on individuals who had proteinuria.

\section{Acknowledgements}

To the staff of Kenyatta National hospital renal unit laboratory, transplant clinic and registry for all the support offered during the study and the kidney donors for accepting to take part in the study.

\section{References}

[1]. Sean J. Barbour, Michael Schachter, Lee Er, Ognjenka and Adeera Levin. A systemic review of ethnic differences in the rate of renal progression in CKD patients. Nephrol Dial Transplant 2010; 25:2422-2430.

[2]. Naicker S. Burden of End-stage renal disease in Sub-Saharan Africa. Ethn Dis. 2009;19:S13-S15

[3]. Evans K, Coresh J, Bash LD, Gary-Webb T, Köttgen A, Carson K, et al. Race differences in access to health care and disparities in incident of chronic kidney disease in the US. Nephrol Dial Transplant 2011;26:899-908

[4]. Ricardo Correa-Rotter. The cost barrier to renal replacement therapy and peritoneal dialysis in developing world. Perit Dial Int.2001;21:S314-317S

[5]. Harris RC, Neilson EG. Toward a unified theory of renal progression. Annu Rev Med. 2006; 57: 365-80

[6]. Otieno L.S., Awori N.W., Bagshawe A.J., Abdulla M.S. and Kyambi J.M. et al. The first kidney transplant in Kenya. East Afr. med. J. 1980; 57:369-373

[7]. Cassidy MJ, Beck RM. Renal functional reserve in live related kidney donors. AM J Kidney Dis. 1988; 11(6):468-72

[8]. Abdu, N Morolo, A Meyers, R Britz, S Naicker. Living kidney donor transplants over a 16 year period in South Africa. Annals of African medicine 2011;10(2):127-131

[9]. Jody A. Charnow. Living Kidney Donors Face Large Risk for CKD. March 19, 2011 Print Article-Renal and Urology News. http://www.renalandurology news.com/living-kidney-donors-face-large-risk-for-ckd/printarticl...

[10]. The JNC -7 Report: JAMA 2003; 289:2560

[11]. WHO. Physical status: the use and interpretation of anthropometry. Report of a WHO expert committee. Who technical report series 854 Geneva: World health organization, 1995

[12]. KDOQI 2002 classification of CKD. Nephrol Dial Transplant Sep 1, 2010; 25:2832-2836

[13]. Siebels M, Theodorakis J, Schmeller N, Corvin S, Mistry-burchardi N, Hillebrand G, et al. Risks and complications in 160 living kidney donors who underwent nephroureterectomy.Nephrol Dial Transplant 2003;18:2648-2654.

[14]. Siragy HM. Translational Nephrology Angiotensin AT1 and AT2 receptors - the battle for health and disease. Nephrol Dial Transplant 2007; 22:3128-3130

[15]. RK Wan, E Spalding, D Winch, K Brown and CC Geddes. Reduced kidney function in living kidney donors. Kidney Int. 2007; $71: 1077$.

[16]. Fourcade J. et al. Risks and complications in living kidney donors. Abstract French Journal, Nephrolgie 2002:23(4)

[17]. Gai M, Giunti S, Lanfranco G, Segoloni GP. Potential risks of living kidney donation- a review. Lancet; 2007:3122-3127. 